AURA ROSALÍA CRUZ ABURTO

UNIVERSIDAD NACIONAL AUTÓNOMA DE MÉXICO

CIUDAD DE MÉXICO, MÉXICO.

AURA@AURACRUZABURTO.COM
Fecha de recepción: 26/01/2021

Fecha de aceptación: 01/06/2021

Cómo citar: Cruz Aburto, A. (2021)

Diseño ontológico transindividual:

Un giro afectivo a la propuesta de Escobar. RChD: creación y pensamiento, 6(10), 01-19. https//doi.org/10.5354/0719-837X.2021.60877

Revista Chilena de Diseño,

RChD: creación y pensamiento

Universidad de Chile

2021, 6(10).

http://rchd.uchile.cl

\section{Diseño ontológico transindividual: un giro afectivo a la propuesta de Escobar}

Transindividual Ontological Design:

an Affective Turn to Escobar's Proposal

Resumen. Frente a la modernidad y sus consecuencias en Diseño, Arturo Escobar ha planteado un desmontaje que revela problemas conceptuales que han producido una atomización de la experiencia. El autor no se ha conformado con este trabajo crítico, sino que ha emprendido una propuesta para retejer lo escindido y restablecer el equilibrio social y ecológico. En el seno mismo de los conceptos que orientan su propuesta se encuentra, sin embargo, un problema. La manera en que articula la idea de autonomía, en función del concepto de autopoiesis, hace muy complicado el establecimiento de una relacionalidad entre comunidades humanas heterogéneas. Esto no es trivial, de cara al problema común que enfrentamos: la supervivencia misma de la vida en el planeta Tierra. El siguiente texto busca abordar una alternativa conceptual que posibilite una auténtica relacionalidad ontológica a través de la introducción de la afectividad, entendida en los términos de la pensadora Suely Rolnik y la propuesta de una ontología del devenir de Gilbert Simondon. A su vez, dicha propuesta permitirá explicar el proceso de conformación, automantenimiento y devenir de los organismos, así como de las comunidades: la individuación servirá de base para un Diseño relacional para la autonomía.

Palabras clave: afectividad, autonomía, Diseño, percepción, relacionalidad, transindividuación

\begin{abstract}
In the face of modernity and its consequences for Design, Arturo Escobar has proposed an analysis that unveils conceptual problems that have produced an atomization of experience. The author was not satisfied by doing critical work, however, and has made a proposal to reweave that which is divided and to reestablish social and ecological equilibrium. There is a problem, however, at the core of the concepts that are fundamental to his proposal. The way he builds the idea of autonomy is based on the concept of autopoiesis, which makes the establishment of the interweaving between heterogeneous communities very difficult. This is not trivial, considering the problem we are facing: the survival of life on Earth. This text seeks to address a conceptual alternative that allows for an authentic ontological relationality, with the introduction of affectivity, as presented by Suely Rolnik's and Simondon's conceptual frame. This proposal will also explain the process of conformation, self-maintenance and especially, the transformation path of organisms as well as communities: the process of individuation.
\end{abstract}

Keywords: affectivity, autonomy, Design, perception, relationality, transindividuation 


\section{Introducción}

La humanidad vive una crisis producto de una forma de existencia insostenible. Las promesas de esta formación histórica han llevado consigo un corolario, hoy catastrófico (Beck, 1998), que comenzó a hacerse visible en tiempos recientes. Unos han llamado a esta era el Antropoceno, otros Capitaloceno. El antropólogo y pensador de Diseño, Arturo Escobar, junto con otros, han identificado el problema en una base más amplia que consiste en el mecanismo de dualismos escindidos propios del pensamiento moderno.

Con base en este fundamento, Escobar (2016) -autor con el que este texto dialoga, debate y trata de conformar un diálogo transformativo- desmonta a la modernidad. Después, da lugar a una contrapropuesta que permite entretejer la vida en un ámbito de horizontalidad y cooperación entre humanos y no humanos. En este contexto, el autor reconoce la necesidad de dar luz a la dimensión política que subyace a la imposición de una ontología sobre otras. En este tenor, detectará que una serie de movimientos sociales (Esteva, 2008) ya han dado forma concreta a una dimensión alternativa: la de la autonomía (Escobar, 2017). De hecho, la fragua de un Diseño autónomo supone una aportación específica de Latinoamérica al Diseño del sur global propuesto por el pensador del Diseño Anthony Fry (2017). Escobar mencionará diversas experiencias de esta autonomía, tales como el Zapatismo en México, la de los Nasca en Colombia, o bien el movimiento del pueblo Mapuche en Chile, de quienes rescata la siguiente declaratoria de autonomía: "Queremos ser nosotros los que diseñemos y controlemos nuestros proyectos de vida" (Rocha, 2015, p. 97).

Aunque Escobar no inventa, sino identifica una práctica de autonomía en curso de acción, en su libro Autonomía y Diseño (2016) busca articular una propuesta teórica que explique y permita complejizar esta idea. Para ello, acuñará una estrategia basada en una serie de supuestos provenientes del enactivismo de los biólogos chilenos Francisco Varela y Humberto Maturana (1980). Frente a lo planteado por Escobar, en este artículo se elaborarán dos objeciones: por un lado, se detectará una contradicción entre autonomía y relacionalidad si queremos pensar en la posibilidad de un entretejido ontológico que haga posible la emergencia de ontologías inéditas más allá de las identidades existentes, o lo que él mismo identifica con el concepto de frontera de Fry (2017). La segunda objeción se hará a la pretensión de que la vía epistémica es por la cual se pueden construir relaciones entre mundos, específicamente a través de la traducción de saberes (Santos, 2009).

Para dar alternativa a dichas objeciones, se plantea la hipótesis del presente trabajo que afirma que no es a partir de la cognición (apoyada en la percepción-acción, proceso de divergencia) como podemos encontrar la vía para comprender a la relacionalidad como fenómeno vital, sino por el proceso de la afectividad que, de acuerdo con lo aquí propuesto, es la base ontológica de toda relacionalidad. Derivado de esto, se explicará que el proceso de la afectividad es crucial en la creación de lo inédito entre dos mundos, que darán lugar a un tercero sin precedentes: una relacionalidad ontológica y no meramente instrumental. De aquí, el objetivo general de este trabajo consiste en plantear una aportación filosófica que permita enriquecer la propuesta teórica de Escobar, para sortear la contradicción que 
guarda su planteamiento entre autonomía y relacionalidad, de tal suerte que puedan derivarse nuevos caminos para la creación de mundos posibles y posibilitantes desde el Diseño.

\section{Nota metodológica}

El presente texto se centra en una crítica y propuesta de ampliacióndesviación a la propuesta teórica que Escobar articula para configurar un concepto de autonomía. Compartimos sin duda sus principios éticopolíticos, pero consideramos que existen problemas conceptuales que pueden solventarse.

Para tales fines, el método utilizado para desentrañar la propuesta del autor consiste en un desmontaje conceptual, basado en el reconocimiento de la composición y de las adscripciones filosóficas de los conceptos utilizados, es decir, de sus genealogías (Foucault, 1988). La crítica aquí presentada, se dirige puntualmente a la base enactivista de la propuesta teórica para referirse a la autonomía y no a los movimientos sociales que ameritan otra clase de tratamiento.

\section{Desarrollo}

\section{El peligro de las escisiones}

Los efectos de la ciencia moderna y, sobre todo, de los supuestos que les sostienen, han cobrado una fuerza que no era concebible en Occidente hasta hace apenas un siglo. Se consideraba a la naturaleza como algo inmutable y separado de nuestro ámbito de acción. Por ello, la ética solía circunscribirse al ámbito de las interacciones humanas interpersonales e inmediatas (Jonas, 1995, p. 16), abrigadas por el mundo antropogénico, aquel mundo común de Hanna Arendt (2009), el mundo de los hombres libres.

Debido a los efectos sistémicos de los avances tecnocientíficos sobre la forma misma de la vida en la Tierra, hoy se plantea la necesidad de que la tecnociencia se cuestione en términos éticos. La ética y la política deben sobrepasar sus límites tradicionales y volverse al ámbito mismo de la ontología.

Es necesario notar que el ámbito del trabajo opera también en el de la necesidad, que tradicionalmente Arendt atribuía meramente a la labor: aquello que denominamos naturaleza, para diferenciarlo de lo meramente humano, en realidad no posee la estabilidad ni la consistencia que Occidente le ha atribuido. Lo que artificialmente hemos separado de su ámbito, nuestro trabajo y nuestra acción (lo político), está entretejido íntimamente en un complejo existencial con la naturaleza por lo cual termina por transformar los cauces mismos de ésta y viceversa. Esto revela que el ámbito valorativopolítico no puede ni debe ser escindido de lo ontológico, nuestro saber implica valoraciones que devienen en formas de hacer que, a su vez, trastocan al ser.

Lo anterior corre en sintonía con la crítica que realiza Escobar (2016) a la modernidad, basada en el riesgo de la atomización de los campos de la experiencia: el saber, el hacer y el ser. El colombiano hace esta crítica a partir de un análisis de la manera en que el pensamiento moderno se ha conformada como un instrumento de dominación. Dicho pensamiento no sólo opera una escisión entre campos de la experiencia que desprenden a su 
saber experto de sus implicaciones ético-políticas, sino que lo hace a través de dualismos en los que se privilegia a uno de los polos, confiriéndole la verdad, la realidad y la racionalidad. De esta manera, el mundo moderno se autoinstituye como el mundo verdadero, real y racional, y desata con ello una serie de injusticias epistémicas y denegaciones ontológicas.

\section{Diseño y modernidad}

De acuerdo con lo expuesto, el pensamiento moderno provee un fundamento común que permite entender que hablamos de una misma formación histórica que comparte una episteme y una ontología específicas, que alimentó en su momento diversas expresiones del Diseño occidental.

Una de las primeras operaciones que se presenta en este pensamiento es la división entre el conocimiento y el ámbito ético-político. Esta escisión se acompaña del dualismo sujeto-objeto a través del cual se reconoce que cada uno de estos polos posee un ámbito exclusivo de actividad. Mientras el conocimiento es el ámbito de la objetividad, la ética (y la política) se constituyen como espacios de la subjetividad y la intersubjetividad. Esta creencia parte de la idea de que la naturaleza es una entidad estable que no es alterada en lo fundamental por las decisiones humanas. Actualmente los efectos de nuestra actividad sobre la Tierra hacen muy difícil sostener esta posición (Jonas, 1995).

Además de la dicotomía sujeto-objeto, la modernidad acuñó otras para caracterizarse. Entre ellas, la dupla mente-cuerpo, condición de posibilidad para la idea de universalidad de aquello que escapa a la contingencia de la carne y del lugar. Diversas expresiones de Diseño se orientarán por estos supuestos con la pretensión de ser capaces de resolver problemas universalmente compartidos.

Finalmente, el pensamiento moderno opera una subsunción: identifica sus elaboraciones epistémicas y ontológicas con la totalidad posible. De esta manera, todo saber con sello moderno adquirirá un estatus privilegiado respecto a otros saberes a los que relegará a mera creencia sin fundamento.

Así, el Diseño fundado en supuestos modernos, se pretenderá objetivo. Gracias a su cientificidad pretenderá resolver problemas claros y distintos, universales, con soluciones liberadas de toda subjetividad. Esto se pensará posible porque la corporeidad y el lugar serán consideradas contingencias superables. Finalmente, dado que el pensamiento moderno supone que su comprensión de la realidad es un espejo de ésta, su diseño será capaz de dar respuestas generalizables y unívocas, de construir un mundo que es real, efectivo y absoluto, para adquirir así una pretendida superioridad epistémica respecto a otras prácticas.

\section{La contrapropuesta de Escobar}

\section{Pluriverso}

Esta autoinstitución del pensamiento moderno como verdadero, real y racional, trae consigo un problema de injusticia y también cancela otras posibilidades que podrían hacer viable la existencia de una mejor manera respecto a la producida por la modernidad. 
Pero, además de las implicaciones pragmáticas que supone no contar con una mayor diversidad de epistemes y ontologías, es un error identificar una sola formación histórica con la existencia misma. Subsumir todo lo existente a un solo mundo es el resultado de confundir conocimiento con el ser mismo. También es problemático considerar que lo que existe está dado de una vez y para siempre, particularmente si participamos de un principio de inmanencia y no de una metafísica (de corte trascendental). Frente a esta actitud ontológica reduccionista e inmutable, Escobar propone retomar la noción de pluriverso, acuñada por William James a principios de siglo XX (Rossi, 2015) que consiste en el entretejimiento de los muchos mundos en un proceso inagotable de transformación. En palabras de Gilbert Simondon (2015), el ser es siempre en devenir.

\section{Ontología relacional}

Otro aspecto fundamental de la crítica de Escobar en Autonomía y Diseño (2016) consiste en la manera que la modernidad constituye su experiencia de mundo a través de la relación sujeto-objeto. Este esquema imposibilita comprender la relación que guardan las existencias entre sí, ya que somete a algunas de ellas -las no humanas e incluso también a ciertos humanos que no han sido considerados sujetos históricamente- a la inactividad, a la pasividad y al sometimiento. De esta manera sólo los considerados sujetos son poseedores de voluntad y capacidad de autodeterminación. Frente a esta distinción, Escobar propone replantear nuestra comprensión ontológica en términos de una red entre singularidades, que no se forma a posteriori de ellas, sino en la que éstas son sus nudos en mutua influencia. A esto llamará ontología relacional y en ella no sólo jugarán un papel todos los seres humanos y los seres vivos, sino también las entidades inertes y, con ellas, los artefactos y nuestras prácticas. Esta red se reconforma constantemente a partir de las tensiones entre sus nodos, no hay primacía de sujeto ante ningún objeto, ni siquiera existe tal escisión.

\section{Diseño ontológico}

La red existencial que caracteriza a esta ontología se conforma de singularidades que crean ontologías concretas en función de su circunstancia corpórea y de lugar, ya que ninguna de ellas resume ni expresa la totalidad de la red que, por otro lado, es indeterminable debido a su constante transmutación.

Esta multiplicidad de ontologías constituye saberes diversos, entre los que no tiene sentido establecer una jerarquización. La práctica del Diseño ontológico supone, además, que toda producción humana nunca es sólo humana, sino que está dada por la interacción de fuerzas concretas en las que se gesta: la del lugar, la de lo humano y lo no humano. Al tiempo que hace, conoce; al tiempo que conoce, hace. Constituye mundo por medio de su acción -conocer, hacer y ser forman un eje de continuidad-. El Diseño configura y da lugar a nuevas direcciones de la existencia: nuevas formas de ser-en-el-mundo (Heidegger, 1986).

Por lo tanto, el Diseño produce objetos, y también genera nuevas formas de interacción. Por ello, es saber, es ético-político y es ontológico. El Diseño ontológico es relacional. La cuestión que emerge para quien suscribe este texto, consiste en cómo es que los muchos mundos permean unos en 
otros, en las formas de operar de los subsistemas de la red y cómo en esta lógica de entretejimiento puede tener lugar la autonomía de los mundos, sin renunciar por ello a la relacionalidad.

\section{Autonomía}

El gran problema de la modernidad, que por sí misma es tan sólo un mundo entre otros, es que busca subsumir toda realidad a sus lógicas. De esta manera, propicia la invisibilización y el sometimiento de otras formas de existencia y tiene por efecto el empobrecimiento y debilitamiento de la vida que actualmente puede evidenciarse en la amenaza a las posibilidades futuras de la existencia misma de nuestra especie, que con su actuar se lleva muchas otras.

Adicionalmente, la lógica moderna parte del dualismo mente-cuerpo, que ha privilegiado a la mente y le ha conferido realidad aparte. Su carácter abstracto presupone universalidad, simplemente porque no está consciente de su historicidad ni de su concreción: de su temporalidad, de su cuerpo, de su lugar. Se realiza una operación de generalización del saber y del ser. Las singularidades -que expresan la infinitud del pluriverso- son invisibilizadas. Se comete el error (o la trampa perversa) de poner una parte por el todo: el mundo moderno en el lugar del caos de Deleuze y Guattari (2001) o el pluriverso de James (1977). Por ello, el problema de la subsunción no es sólo un problema de injusticia ético-política (en que se niega el derecho de otros a definir la propia existencia); sino también ontológico.

En esta tónica, la autonomía debe ser entendida como la defensa que emprenden los otros mundos por su existencia y por su derecho a autodeterminarse frente al mundo moderno, el cual los ha invisibilizado y condenado a la servidumbre y/o a la extinción. Se incluyen no solo las comunidades humanas sino a las comunidades complejas que conforman con lo no humano. De estas realidades históricas concretas emerge la propuesta de autonomía, como una manera de resistir y reexistir.

Estas prácticas de autodeterminación también implican la condición de posibilidad de la diversidad ontológica y epistémica, que puede ser garante de que este planeta tenga futuro. La autonomía, aunque nace como resistencia y reexistencia de estos pueblos, es vital para la transformación general del mundo para dar cara a los efectos del Capitaloceno. Al ritmo que vamos, de no desarrollar una transformación profunda que incluya a las sociedades occidentales, el futuro no es nada promisorio para nadie. La gran pregunta que emerge es: ¿cómo mantener la capacidad de la relación y la transmutación creativa entre mundos? ¿Cómo lograr mantenerse en el entretejido del mundo (pluriverso) al mismo tiempo que se ejerce la autonomía? ¿Cómo evitar nuevas subsunciones? Es absolutamente debido que cada mundo reclame su derecho a la autonomía. Pero deberá ocurrir en un horizonte de relacionalidad, en una copertenencia al pluriverso.

\section{Enactivismo, autopoiesis y autonomía}

Un concepto clave que utiliza Escobar (2016) para fundamentar teóricamente las resistencias ontológicas es el de autonomía. Este pensador lo explicita a través de la noción de autopoiesis, proveniente de la propuesta enactivista de Varela y Maturana (1980). 
La enacción es una manera de entender la cognición en la que confluyen la percepción y la acción como dos caras de la misma moneda. Percibimos (y por tanto conocemos) el mundo al tiempo que actuamos (hacemos) sobre él (Maturana \& Varela, 1987). El mundo se rehace continuamente: los agentes actuamos sobre él y lo transformamos al tiempo que nos moldea. A partir de esta noción es posible ver que mente, cuerpo y mundo no están separados (Ingold, 2011).

En el marco de la enacción, Varela y Maturana (1987) proponen que, si bien existe entretejimiento entre agente y mundo, los agentes están a la vez entretejidos y diferenciados del entorno. Tienen la capacidad de autoproducirse continuamente sobre la base del mecanismo de autopoiesis. Una característica importante de esta facultad de autoproducción radica en que su referencia es el agente mismo. Los autores plantean que la operación fundamental de todos los seres vivos reside en la capacidad de organización autónoma, que permite actuar efectivamente en su entorno para mantener su existencia y para desplegar su desarrollo. La organización consiste en un sistema interno de relaciones que, de perderse, produce la muerte. Para que este sistema se mantenga, se deben respetar ciertos criterios de conservación de la organización que establecerán los límites y formas de relación con el entorno: su acoplamiento estructural. En este acoplamiento la unidad autopoiética (organismo) determina la relación, no el entorno. Hasta aquí, el enactivismo de Maturana y Varela, que sirve a Escobar para caracterizar la noción de autonomía que va desde lo biológico hasta lo social, da al organismo el papel rector del mantenimiento de la vida y de la dirección de las transformaciones.

Lo anterior produce un doble cuestionamiento. En primer lugar, en esta caracterización aún existe un resabio de la vieja dualidad sujeto-objeto, ya que se confiere poder de determinación a un solo lado de la experiencia viva (la unidad autopoiética), para dejar al entorno en una posición secundaria. Por otro lado, hay que cuestionarnos si es deseable buscar el mantenimiento de una organización por siempre, sin aceptar las mutaciones que son también producto del devenir de la vida, que suponen aparición y desaparición de cartografías existenciales. Es comprensible que una comunidad persiga el mantenimiento de su existencia y de su autonomía, pero también es normal que mute hasta convertirse en otra. Esto no tiene por qué ser necesariamente un problema, a menos que se dé por efecto de la dominación de un sistema heterónomo. A continuación, veremos qué se puede pensar al respecto de cada una de estas objeciones y qué puede proponerse de manera alternativa para poder dar cauce a una autonomía auténticamente relacional.

\section{Objeciones a Escobar}

\section{De las representaciones a las affordances}

La psicología ecológica tiene por objetivo dar cuenta de la cognición a través del concepto de affordances. La piedra fundacional de esta propuesta (Gibson, 1979) descansa en la diferenciación entre mundo físico y ambiente animal. Desde el mundo físico, el espacio se entiende en términos geométricos; en el caso del ambiente será entendido en términos de locomoción y percepción (puntos de observación, lugares donde un animal 
1. Forma de vida es entendido como las maneras

típicas de hacer ciertas cosas y las formas de vivir más o menos constantes que se pueden observar en grupos de animales (Wittgenstein, 1953). coloca sus órganos sensorios, sus pautas de movimiento). En cuanto al tiempo, en el caso del ambiente se trata de eventos a través de los cuales las cosas se transforman y persisten. El ambiente no es el mundo físico, sino aquel que tiene sentido para el agente. No se trata de una entidad separada, sino de un subconjunto del mundo físico considerado en función de las ventajas que da al agente.

Mientras que el mundo físico es sustantivamente no significativo, el ambiente contiene affordances. Las affordances resultan de la articulación entre aspectos ambientales (socio-materiales) y las destrezas y habilidades disponibles en una forma de vida ${ }^{1}$. Gibson (1979) defiende la idea de que éstas no son propiedades ni objetivas ni subjetivas, sino ambas. Lo anterior se explica por la introducción del correlato animal (agente) que, aunado al correlato material (información del ambiente), dan lugar al sistema relacional emergente llamado affordance.

Ahora bien, mientras la representación supone un trabajo de cómputo mental necesario para relacionarse con el mundo (y que se separa de él), las affordances implican una percepción directa del mundo, no mediada, que además ocurre en función de las posibilidades de acción y que se actualiza por ellas. Resulta innecesario construir y manipular representaciones, dado que el ambiente ya contiene a las affordances que se actualizarán en función de las destrezas y habilidades del agente. No hay representaciones interiores, hay mundo significativo. La experiencia significativa es la acción misma que tiene lugar cuando el agente enfrenta sus destrezas y habilidades (posibilitadas por una forma de vida) a la información del ambiente que da por resultado la emergencia de ciertas affordances.

\section{La confrontación entre psicología ecológica y enactivismo}

De acuerdo con Baggs y Chemero (2018), la psicología ecológica se enfoca en la naturaleza del ambiente, mientras que el enactivismo se enfoca en el organismo como agente. Si aceptamos que las affordances son de naturaleza interactiva, no propiedades sino relaciones, ellos proponen que la combinación de ambas perspectivas permite completar la imagen de la cognición al articular la historia del agente con la del ambiente. El punto de coincidencia descansa en la affordance. La psicología ecológica tiene su punto de partida en el ambiente (significativo para el agente), el enactivismo tiene como punto de partida al agente individual.

Pese al optimismo conciliador de Baggs y Chemero (2018), es importante destacar que, en la propuesta de Maturana y Varela (1980) se atribuye un carácter determinante al agente sobre el entorno. Las affordances de la psicología ecológica plantean una relacionalidad más franca que la propuesta de la unidad autopoiética del enactivismo, al proponer una categoría relacional que no es ni objetiva ni subjetiva y que cobra sentido en el encuentro del agente y el ambiente. Ahí cobra realidad la vida y se reconoce que el ambiente también contiene posibilidades singulares y no sólo el organismo posee destrezas. Las affordances nacen de la interacción entorno-agente. No se deben meramente a un código preinstalado que dicta la organización de un organismo. 
De la autopoiesis a la antropofagia crítica

A partir de la objeción hecha a la noción de autonomía que propone Escobar, la psicología ecológica ofrece la alternativa de las affordances. Sin embargo, aunque esta categoría consigue reestablecer la horizontalidad entre agente y ambiente, no queda claro cómo podría ser posible una relacionalidad entre agentes diversos con mundos perceptivos diferentes. ¿Dónde reside la condición de posibilidad del pluriverso?

Además, desde el punto de vista normativo hay que cuestionarnos si es conveniente perseguir el mantenimiento de una organización por siempre (esencialismo), con el corolario de la no aceptación de las mutaciones (la operación creativa de la vida misma). Aunque es comprensible que una comunidad persiga el mantenimiento de su existencia y de su autonomía -sobre todo si ha vivido sometida e invisibilizada-, también puede ser deseable, bajo condiciones de horizontalidad, que las comunidades muten, incluso hasta convertirse en otras. Combatir la heteronomía producto de la dominación de una forma de vida sobre otra, no debe suponer necesariamente la eliminación de la transmutación de formas de vida diversas entre sí, porque esto no ocurre necesariamente por dominación, sino también por efecto del entretejimiento mismo de la vida. Esta transmutación mutua, es la posibilidad de la idea de frontera que propone Fry (2017): "el espacio intermedio de pensamiento y acción basado en actos políticos y pragmáticos de apropiación de tipo bricolaje [...] donde se intercambia información, se traducen entre sí los diversos mundos y donde la solidaridad y la amistad se forjan" (Fry, 2017, p. 12).

Es necesario plantear otro sentido de autonomía distinto al de autopoiesis, así como es conveniente esclarecer las condiciones de posibilidad de lo común que conforma al pluriverso. Para comprender la actividad creativa de la existencia más allá de todo esencialismo cultural, para hacer asequible el establecimiento de la relación entre mundos diversos sin perder la posibilidad de la autonomía de las singularidades se acudirá a la ontología de Simondon (2015) denominada individuación, junto con la noción de paradoja sensible propuesta por Rolnik (2005).

De la autonomía a la singularidad relacional de las fuerzas Como se ha discutido, existe un problema en la propuesta de autopoiesis de Maturana y Varela debido a que centraliza la actividad en el organismo y deja en un lugar secundario al entorno. Por otro lado, aunque la psicología ecológica logra sortear esta dificultad, no consigue explicar cómo suceden los encuentros, entrelazamientos e incluso intercambios entre los muchos mundos, de tal suerte que no sólo signifiquen la instrumentalización de unos por otros (colonizaciones), sino la posibilidad de transmutarse entre sí. Lo que no logra responderse a partir de estas propuestas cognitivistas es qué es y cómo es posible el pluriverso².

A continuación, se presentará el corpus de la ontología del ser en devenir de Simondon (2015) conocida como la individuación, que puede dar lugar a la consolidación de un Diseño ontológico plenamente relacional como el que Escobar persigue, y que no tiene como base del entretejimiento
2. A decir de William James, un mundo pluralista

e inacabado, uno y múltiple, en permanente recomposición, no hecho de otra cosa que de diferencias, experiencias situadas, conexiones parciales, novedades e historias múltiples (Savransky, 2019). 
fundamental un trabajo de orden epistémico -como supone la traducción de saberes de Santos (2009) (aunque tampoco tiene que ser descartado)sino la apelación a la afectividad.

\section{Individuación}

Simondon (2015) detectó un problema de origen en la concepción del ser de la filosofía (occidental), que le ha llevado a diversos callejones sin salida: el esquema hilemórfico desdobla al ser en una dualidad forma-materia que es atribuido a un poder de transformación exclusivamente humano; por otro lado, plantea que el ser es unidad indivisible e idéntica a sí misma.

Uno de los grandes problemas del esquema hilemórfico es que da lugar a la distinción sujeto-objeto, tanto como mente-cuerpo, donde un lado es activo (sujeto-mente-forma) y el otro es la mera receptividad (objeto-cuerpomateria), una división que promueve la dominación. En términos ontológicos no permite comprender la relación de mutua transformación entre agente y medio ambiente, y en la relación del individuo viviente tampoco posibilita la comprensión de éste como agente, medio y elemento de su propia individuación. El hilemorfismo también supone que forma y materia son anteriores al devenir y que en ellos se identifica el ser. Simondon (2015), por su parte, señala que el ser no sólo no es lo opuesto al devenir, sino que por definición es ser en devenir. El ser siempre está polarizado: no está en un estado estable sino metaestable, un equilibrio frágil, a punto de cambiar.

\section{Las fases de la individuación}

El ser no es una esencia detenida, ni una consistencia clara, ni siquiera dual (forma-materia). Es proceso de diferenciación con diferentes fases discontinuas entre sí. Estas fases tienen lugar cuando el individuo ya no resuelve el conflicto en su estado actual de metaestabilidad debido a su sobresaturación, y entonces se desfasa de sí mismo y deviene en un proceso de transducción (devenir otro) que llegará a ser irreversible a partir de cierto umbral. Las diversas fases que identifica Simondon (2015) son la física, la biológica, la psíquica, la social y, en su trabajo acerca de los objetos técnicos, la concretización (individuación técnica).

Es importante señalar que entre estas fases existe una interacción entre lo discontinuo y lo continuo, entre el individuo y su medio asociado. Aunque todas las fases están vinculadas, también les separan cambios repentinos de umbral. No necesariamente implican una mayor complejidad sino una alteración específica que responde a la resolución de un conflicto que tiene lugar por un plus (la dimensión creativa del ser). Por ejemplo, el salto de la individuación física a la biológica no supone que el individuo vivo sea más complejo que el físico, sino que tiene lugar por una ralentización tal del proceso de individuación que emerge un ser con capacidad de individuación sostenida en función de su propio automantenimiento.

La individuación es un régimen energético entre individuo y medio asociado que en conjunto conforman una realidad completa anterior. A partir de sus interacciones se sobreviene el advenimiento de nuevas individuaciones sucesivas, nuevos despliegues (no como en el planteamiento de Maturana y Varela, donde la acción es determinada por el agente). Cada uno de estos 
despliegues sólo será una posibilidad del devenir que se ha actualizado a partir del sistema metaestable de origen y que dará lugar a un nuevo individuo con su respectivo medio asociado.

\section{Información y significación}

Las interacciones del individuo y su medio asociado pueden ser entendidas en la medida en que la información las vincula. La información debe ser entendida como el reconocimiento de los potenciales implícitos en el individuo y su medio asociado en tanto sistema en estado metaestable: sus posibilidades múltiples de devenir. Un ejemplo que Simondon propone para dar cuenta de esto es cuando una persona trabaja la madera con una garlopa. El trabajo realizado no se basta de haber aprendido ciertos gestos técnicos, sino también de la captación sensorial de las posibilidades intrínsecas del trozo de madera, sus potenciales, su información. Estas posibilidades se detectarán como señales a través y por la garlopa en la medida en que ésta permita transmitirlas $y$, al ser sentidas y percibidas por quien trabaja la madera adoptarán una significación de lo que puede ser realizado).

En el caso del ser viviente, la información adquiere una dimensión particular pues, dada la naturaleza de la individuación biológica, no sólo se almacena y transfiere. Al cabo de cada una de las individuaciones sucesivas se amplifica, se diversifica, se actualiza de acuerdo con las incompatibilidades que se encuentran a lo largo del desarrollo, y da lugar a nuevas resoluciones o significaciones que, de nuevo, se transferirán como información para el siguiente despliegue. Hay aprendizaje que se hereda y se amplifica. Este concepto de información permite entender las operaciones de las comunidades biológicas en su proceso de transmisión dinámica de información en la cual, a este nivel, el individuo ${ }^{3}$ mantiene una relación de diferenciación más o menos estable entre sí mismo y su medio asociado. Sin embargo, es un error pensar que este proceso de individuación biológica es totalmente transferible a los siguientes umbrales que Simondon ha reconocido como individuación psíquica y social.

Debido a lo anterior, un problema de la propuesta enactivista, que hereda Escobar, es que extiende la lógica de la individuación biológica al ámbito de lo social. Si bien, como Escobar, asistimos a la idea de continuidad entre naturaleza y cultura, esto no debería suponer la subsunción de lo social a las lógicas biológicas. La riqueza de la propuesta simondoniana radica en el reconocimiento del proceso de individuación como un proceso omniabarcante del ser, y también de sus diversas fases, entre las que se presenta una discontinuidad caracterizada por saltos cuánticos.

\section{Del entorno como forma al entorno como fuerza}

De acuerdo con Escobar (2016), en resonancia con Santos (2009), la manera de entretejer a las comunidades, cada una de las cuales posee una ontología singular, es a través de la traducción de saberes: un proceso fundamentalmente epistémico. En lo que sigue se defenderá que, si bien poner en acción la traducción (entre saberes) puede ayudar a dialogar, también puede alcanzar el espacio de la inconmensurabilidad (Kuhn, 1989).
3. Se debe tener presente que no es el individuo moderno sino el agente $u$ organismo, la comunidad, en lenguaje simondoniano. 
El ámbito cognitivo supone la configuración de mundos particulares. Cada agente, así sea un colectivo, segmenta y modela su propio mundo de acuerdo con sus particulares procesos de percepción y acción. La cognición da lugar al mundo propio, es un proceso de diferenciación, no de entrelazamiento. Pretender encontrar por medio de la cognición el principio de lo común es un sinsentido, y puede dar lugar a la dominación por la vía de la subsunción (su proceso fundamental), con la ilusión de haber encontrado conceptos universales. Abundemos en la naturaleza del proceso de la percepción-acción para mayor claridad.

\section{Percepción-acción}

De acuerdo con Simondon existen dos procesos que deben diferenciarse: la percepción-acción y la afectividad. El primero permite la captación de la actividad del mundo gracias al proceso cognitivo de la percepción, cuya operación fundamental consiste en integrar los datos de los sentidos en un objeto de conocimiento.

La percepción no completa el proceso del todo. Si bien reúne términos que no coinciden, y de esta manera da lugar a ciertas significaciones, se trata de descubrimientos parciales entre los que aún persistirán conjuntos diversos. Entonces se pondrá en escena su par, la acción -actualización de la percepción- la cual permitirá que la pluralidad de conjuntos devenga sistema, ya que operará las transformaciones necesarias en el entorno para alcanzar la coincidencia.

Percepción y acción son los dos términos de una misma serie transductiva. Esta manera de comprender percepción y acción supone al agente individuándose a través de ellas. La actividad percepto-activa permite que la información sea capturada, conservada y amplificada en relación con el medio asociado. A su vez, es producto de la integración de los mundos perceptivos en unidad de acción. La percepción-acción es un proceso que se realiza en el comercio entre interioridad y exterioridad del ser viviente (lo que observan las ciencias cognitivas).

\section{Afectividad}

Por otra parte, en la interioridad del ser viviente existen procesos que permiten su automantenimiento y su autotransformación. El automantenimiento es semejante a la autopoiesis de Maturana y Varela (1980), sin embargo, en la propuesta de Simondon (2015) se enuncia una idea ausente en la de los chilenos: existe resonancia entre interioridad y exterioridad, pues comparten un origen preindividual.

Dicha resonancia, posibilitada por dicho origen compartido, establece una relación entre interioridad y exterioridad más allá de lo instrumental y de cualquier jerarquía. No sólo la interioridad (el individuo) organizará y creará mundo por medio de la percepción-acción. La propia interioridad se autotransformará por efecto de su resonancia con el medio asociado. Como en el caso de la autopoiesis, hay un acto de creación de sí, pero este acto de creación no está dirigido meramente por lo que es propio del individuo. Es posibilitado por lo que hay de común con su medio asociado, por lo preindividual. Es importante comprender que interioridad no es meramente estar dentro, sino estar del lado de la cara interna de la membrana que separa del, y une con, el medio asociado. 
La relación intrínseco-extrínseco que presupone la actividad de percepciónacción permite pensar la actividad de organización como resolución contra la entropía y dar lugar a la emergencia del mundo. La relación del ser viviente consigo mismo, en el interior de su proceso homeostático, es el mundo de la afectividad-emoción, y supone la transducción (transformación radical) del individuo a partir de su encuentro con el mundo. Contrario a ser pura interioridad, la afectividad-emoción es el supuesto de la transducción entre estos dos lados de la membrana topológica. Supone el advenimiento de lo que es exterior en interior y viceversa, integración y diferenciación por el proceso mismo de individuación que se explica como efecto de la resonancia entre ellos.

En síntesis, la percepción-acción es la actividad de construir un mundo, un territorio. La afectividad-emoción corresponde a la mutación a partir de la transducción entre individuo y medio asociado. Ninguno de los dos quedará igual después de esta experiencia: mutuación dirá el colectivo Diseño Detonante (Cruz, 2018). En el panorama de la afectividad ya no se trata de organizar y dar forma al mundo (como en la percepción-acción), sino de transmutarse con él para dar lugar a una nueva individuación (y un nuevo medio asociado). A diferencia de la acción y de la representación, la afectividad y la emotividad poseen un carácter transductivo que les hace el espacio del devenir otro. Es el ámbito donde el conflicto se instaura por el advenimiento de una emoción. Permite el autocuestionamiento y el cuestionamiento de la relación con el mundo y el individuo mismo.

En el ámbito de la afectividad y la emotividad somos atravesados y devenimos otros: ahí es posible conformar nuevas comuniones (y no en la traducción de saberes por sí misma), y se expresa la posibilidad de transducción de la multiplicidad que somos nosotros mismos y nuestros potenciales (ecología mental de Guattari (1996), de la mutiplicidad que es lo otro (ecología ambiental (Guattari, 1996)), y la multiplicidad que es el otro (ecología social).

La paradoja sensible: percepción y afectividad

Suely Rolnik, pensadora brasileña, hace una diferenciación muy importante en las dos formas de operación de la sensibilidad que hemos descrito desde los planteamientos de Simondon (la percepción-acción y la afectividad-emoción).

La sensibilidad, ya sea percepción o afectividad, supone "conocer y relacionarse con la alteridad del mundo" (Rolnik, 2006, p. 185). En este proceso, "la materia-mundo es aprehendida como un conjunto de formas o como un campo de fuerzas" (pp. 185-186). Se aprehende por un proceso de percepción (forma), o bien por otro de afectividad (fuerza). En el primer caso, el ejercicio se caracteriza por ser extensivo u objetivante y, en el segundo, se trata de uno intensivo o vibrátil.

La percepción se realiza al proyectar un sentido a una forma, lo cual genera una reducción del mundo (esquemas cognitivos, representaciones, signos). En el caso de la afectividad (sensación para Rolnik) acontece el encuentro de una singularidad con "la presencia viva del otro" (Rolnik, 2006, p.186), con su fuerza. Dicha singularidad otra y el individuo resuenan, tienen un efecto uno sobre la otra y viceversa ${ }^{4}$. Este encuentro de fuerzas vitales, que a veces son de debilitamiento y otras de potenciación, no puede reducirse ni represen-
4. Cuando hablamos del juicio estético kantiano, por ejemplo, es cuando hablamos de afecto en tanto no se trata de describir el mundo, sino de captar cómo éste me afecta, cómo tiene efectos sobre mí. 
tarse. Solo puede expresarse como "una obra de arte, pero también un modo de ser, de sentir o de pensar, una forma de sociabilidad, un territorio de existencia, etc." (Rolnik, 2006, p. 186).

La percepción procede por construcción de formas estereotípicas de comprensión del mundo: es el ámbito del hábito, la costumbre, de la normalidad. Cuando se alcanza un punto donde el individuo y sus esquemas se han vuelto inoperantes (sobresaturación del sistema), no es por su vía que se abre paso una transducción. Es la vía de la afectividad la que lo permite.

[...] La paradoja, a la larga, pone en cuestión las formas corrientes de la realidad cuando estas se tornan un obstáculo para la integración de nuevas conexiones del deseo que provocan la emergencia de un nuevo bloque de sensaciones. Esas formas corrientes [...] se vacían de vitalidad y pierden sentido. Una crisis de subjetividad se instala ejerciendo presión, generando sentimientos de asombro y miedo, causando vértigo.

[...] El sentimiento de asombro y miedo obliga a la expresión de una nueva configuración de existencia, a una nueva figuración de uno mismo, del mundo y de las relaciones entre estos; es eso lo que moviliza el poder de creación (el afecto artístico). El mismo sentimiento también obliga a actuar de tal manera que la nueva configuración puede afirmarse en la existencia e inscribirse dentro del mapa vigente como una realidad compartida, sin la que el proceso no puede completarse; eso es lo que moviliza el poder de acción (el afecto político [...]). (Rolnik, 2006, p. 187)

Por lo anterior, la afectividad es el proceso que vehicula la relacionalidad al interior de cada subsistema (individuo-medio asociado) y en el encuentro de subsistemas que habrán de trastocarse en su encuentro afectivo (encuentro vivo de ontologías).

\section{Un nuevo sentido de autonomía}

Como consecuencia, se propone que la autonomía radica en la condición de posibilidad de los procesos de individuación, siempre singulares, sin olvidar que tienen una doble faz: la singularización-forma, que podemos llamar territorialización (Deleuze y Guattari, 2002), que se realiza por la vía de la percepción-acción; y la afectividad -en lenguaje de Deleuze y Guattari, un proceso de desterritorialización- cuando la organización actual del individuo ya no es operante y se ha hecho necesario promover un proceso de transducción (que involucra, a su vez, otros individuos, físicos, biológicos, psíquicos, sociales).

En síntesis, la autonomía implica la paradoja sensible. Por un lado, está el proceso de construcción del mundo singular a partir de la percepción-forma, que consiste en hacer mundo bajo la organización ya dada del organismo que posee su propia estructura de sentido, de identidad. Por otra parte, en tiempos de sobresaturación del sistema individuo-medio asociado, la autonomía demandará un proceso de transducción entre su propia consistencia (interioridad) y exterioridad (que pueden ser también los otros mundos, las otras ontologías). Ocurre una transformación y se da lugar a la creación de individuaciones (mundos) que permiten que la vida continúe. 
Relacionalidad como transindividuación

En todo proceso de individuación siempre sigue la emergencia de un nuevo individuo acompañado del medio asociado correspondiente. El medio asociado es todo aquello del individuo anterior que no ha sido retomado por la nueva individuación pero que le acompaña y con el que conforma una unidad en devenir. A toda individuación siempre le acompaña un resto de lo preindividual.

Lo preindividual no sólo es huella del origen, es también la posibilidad del porvenir, porque es símbolo. Según Simondon (2015) lo preindividual es un trozo del origen que cada uno tiene. Es lo que permite devenir juntos, independientemente de nuestras individuaciones particulares. El paso de individuación entre individuaciones no se da debido al individuo mismo, sino a la posibilidad de conjunción por los símbolos que se encuentran: la carga de preindividualidad en cada uno. Este es el proceso de lo transindividual.

La experiencia transindividual supone la constitución de lo colectivo; es el proceso de auténtica cohesión social. Supone algo más que la mera interconexión entre individuos, que sería un proceso interindividual ${ }^{5}$. Un suceso transindividual no se detona por las características de las individuaciones singulares sino por la carga preindividual que resta en cada una de ellas. Además, el proceso transforma a los individuos en ese régimen de transindividuación sin que eso implique que se conviertan en una masa totalizante. La relación entre individuo y lo transindividual no es una de subsunción del primero al segundo sino de una superación por prolongación (Simondon, 2015). El individuo no se borra como singularidad, se transforma a través de una amplificación de síque nunca alcanzaría solo.

En este sentido, la transindividuación no se fundamenta en las identidades sino en una resonancia mucho más primitiva, multiplicadora. Este proceso de transindividuación deviene por el cuestionamiento hecho por el prójimo, e implica a un individuo que se ha dispuesto a ser puesto en entredicho. Esta experiencia implica el advenimiento de una emoción que revela una contradicción afectiva. Hay algo en uno que no es identificado con el devenir propio, con la identidad (Simondon, 2015, p. 468). Este problema solamente podrá ser resuelto cuando, en el encuentro de cargas preindividuales, emerja esta segunda individuación que implicará la elección de una significación compartida con otros que no es exterioridad, sino rebasamiento del individuo. ¿La relacionalidad es este rebasamiento!

Por ello, para la formación de un común con otros mundos no es necesario recurrir a nuestras bases identitarias (en donde habitan nuestros saberes). El ámbito de emergencia de lo común no está en hacerse parecido, ni en subsumirse a una de las lógicas rivales, sino en encontrar aquello que en nosotros aún no está determinado, aquello que revela contradicción afectiva, y que sólo en el encuentro performativo con otro se resolverá. Este proceso no nos dejará siendo los mismos, pero tampoco seremos devorados en la heteronomía. Siendo singulares, podremos entretejernos con otros.
5. Es muy importante distinguir entre la conformación de lo colectivo de la mera conformación de un grupo de acción. Este segundo grupo no se puede suponer una auténtica individuación de grupo, sino una mera reunión de individuos que acoplan sus funciones, como podrían ser los grupos conformados por la división del trabajo.

\section{Resultados: hacia el diseño ontológico transindividual}


En los párrafos precedentes se ha planteado la necesidad de incorporar la afectividad al modelo de Escobar. Esto requiere ir más allá de decir que los sentires no pueden ser separados del pensamiento. Hay que hacer una ontología de la afectividad. A través de un marco teórico, apoyado en Simondon y Rolnik, se ha dejado en claro qué comporta la afectividad y por qué a partir de esta noción se puede catalizar una auténtica relacionalidad. Este entretejimiento de los muchos mundos no es trivial cuando sabemos que estamos frente a una crisis antropogénica de escala global.

No se trata de compartir nociones, ni de afecciones específicas (ya que esto implica que el afecto se ha reducido a forma), sino de entender al otro y a lo otro como una singularidad, es decir como un cuerpo vibrátil, un campo de fuerzas en sí mismo, no como un objeto para entender ni para manipular. En el campo de la afectividad-que siempre es performativo y no representacional (Rolnik, 2006)- es donde puede cobrar lugar un proceso transindividual que no sólo extienda las capacidades del individuo -que es tratar a lo otro instrumentalmente-, sino que le articule con lo otro en una nueva entidad: una comunidad.

La afectividad es la condición de posibilidad para configurar un auténtico Diseño ontológico relacional. Pensar en el Diseño ontológico implica que lo que se diseña irrumpe en la existencia y la modifica (Winograd y Flores, 1989) y que existen tantas realidades como acciones porque la realidad no es, sino que siempre está siendo (James, 1977). Si queremos pensar en su dimensión relacional entre mundos (dimensión ontológica-política), la vía de la traducción epistémica queda corta. Permite articular acuerdos, pero no consigue un auténtico entretejimiento. En resumidas cuentas, el Diseño ontológico relacional debe pasar por los afectos.

\section{Afectividad entre lo humano y lo no humano}

Pensar en un Diseño ontológico transindividual implicará que el mundo artefactual no sólo resuelve problemáticas para adecuar mundo y darle forma para servirnos de él (percepción-acción), sino como dice Vilém Flusser (1999), también articula o desarticula relaciones intersubjetivas o, en nuestro nuevo lenguaje, intersingulares. Por ello, el Diseño ontológico transindividual debe operar no sólo como producción de formas operativas (Diseño como función), sino de condiciones de posibilidad para la relacionalidad entre singularidades donde se incluyan, como dice Escobar (2016), humanos y no humanos. Esta práctica contribuirá a visibilizar las alteridades condenadas a la no existencia, al fungir como detonador afectivo entre lo que escapa a nuestro mundo preestablecido.

Se podrá objetar que esto ya sucede en diversas culturas no modernas. También que más bien habría que dejarles existir en paz. Todo eso es cierto, sin embargo, no permite responder las siguientes inquietudes. ¿Qué es lo que tenemos que hacer nosotros (que habitamos un mundo de prescripciones modernas) para transformar nuestra forma de existencia? ¿Cómo podríamos configurar frentes comunes con las resistencias de los pueblos que han mantenido su singularidad a base de lucha, sin caer en subsunciones? Vivir una transducción con esos pueblos en resistencia es una fuente de infinitas posibilidades, pero eso no debe significar injusticas epistémicas 
y hermenéuticas, ni tampoco podemos pretender convertirnos en ellos. Tenemos nuestra propia biografía con sus propios potenciales. ¿Es posible fundar una ontología y una ética en común para el florecimiento de la vida?

\section{Afectividad entre mundos}

Escobar ha planteado la idea de pluriverso, que puede entenderse al retomar del Zapatismo la idea de "Un mundo donde quepan muchos mundos". En este sentido, el pluriverso supone una irreductibilidad de la existencia que se diversifica y constituye ontologías específicas relativas a las comunidades que habitamos la Tierra. Para robustecer la resistencia de las comunidades que viven en esta lucha con los procesos de colonización occidental (moderna), el pensador colombiano propone una manera de entender la autonomía en tanto facultad de autoproducción (autopoiesis) de los pueblos. Este concepto dicta una preeminencia del subsistema (cultura) sobre lo que le es externo: puede optar por adoptar herramientas, instrumentos, prácticas del mundo heterónomo, aunque siempre mantiene su lógica interna porque esta le permite subsistir en tanto es el organismo que es: en eso radica la autonomía enactivista, en el reconocimiento de esa lógica interna, núcleo de su forma de existencia. Sin embargo, esta noción de autonomía presenta un peligro puesto que puede llevar fácilmente al esencialismo cultural -lo cual complica la comprensión y la práctica de las transformaciones que obviamente deberán ser las determinadas por la propia comunidad- $y$, sobre todo, dificulta la posibilidad de entretejer una comunidad de comunidades (la multitud de Hardt y Negri, 2004) más allá de las simples articulaciones estratégicas que nos dé oportunidad de compartir objetivos comunes, aunque cosmovisiones diferentes, frente al gran problema del del Capitaloceno.

Frente al problema descrito se ha planteado un nuevo marco ontológico, donde la carga preindividual, que se despliega por el proceso de la afectividad, moviliza la transducción: transformarse a partir del encuentro con la alteridad y viceversa (donde ninguna de las lógicas precedentes dominará) y donde, la última fase del proceso de individuación, la transindividuación, nos coloca en la posibilidad de entretejernos entre individuos para formar una nueva colectividad sin que eso implique que seamos subsumidos en una masa homogénea. La autonomía, en este marco, radica en desplegar nuestra individuación en toda su potencia lo cual necesariamente alcanza su dimensión más potente en la fase de lo transindividual: no se es autónomo en la clausura.

En este último sentido, una propuesta de Diseño ontológico transindividual no supone la creación de un Diseño internacionalmente compartido (como soñó la modernidad), ni meramente una multiplicidad de Diseños que habitan diversas ontologías. Tampoco se trata de una hibridación entre técnicas, materiales y formas tradicionales aplicadas a una forma de vida moderna ${ }^{6}$ ya que, en ese caso, lo que ocurre es que sólo se instrumentaliza un saber tradicional, pero prevalece una ética y ontología moderna (Cruz \& Cortés, 2020). De lo que se trata es de dar sitio a la experiencia transindividual en el seno del Diseño ontológico: hacer posible la transducción entre Diseños, es decir, hibridaciones ontológico-afectivas -y no meramente epistémicas ni instrumentales- para alcanzar nuevas formas de existencia que creen condiciones de posibilidad para que la vida en esta Tierra siga siendo posible.
6. Una manera de ejemplificar esto puede pen-

sarse con base en la crítica que Cortés y la autora de este texto han hecho respecto a las prácticas de canibalización del Diseño moderno de saberes tradicionales bajo el supuesto argumento de una hibridación de mundos. Lo que se ha hecho es extraer e instrumentalizar técnicas artesanales, al servicio de una ontología moderna. El ejemplo que se ha presentado en el artículo referido apunta las técnicas, formas y materiales artesanales que la diseñadora Clara Porset introdujo en su Diseño de mobiliario que mantuvo unos supuestos de prácticas intersubjetivas modernas (Cruz \& Cortés, 2020). 
El objetivo general del presente artículo fue el desarrollo de una aportación filosófica que enriqueciera la propuesta de Escobar para sortear la contradicción que guarda su planteamiento teórico entre autonomía y relacionalidad, para que de aquí se puedan derivar nuevos caminos para la creación de mundos posibles y posibilitantes desde el Diseño.

La propuesta vertida en este texto se distancia de lo que se ha construido previamente (incluyo la propuesta de Escobar), que suele basarse en las ciencias cognitivas actuales que privilegian los procesos de percepción-acción y dejan al de la afectividad-emoción al margen. En dichas propuestas, aunque se mencione continuamente que el sentir es importante, no se le caracteriza ontológicamente y no se dimensiona su papel en la existencia. En ese sentido, este texto consiguió diferenciar los procesos ontológicos de percepción-acción y afectividad y pudo dar cuenta del potencial vinculante que tiene el segundo respecto del primero (básicamente diferenciador). Se redefinió la autonomía teóricamente concebida por Escobar (2016), en su doble aspecto: el de capacidad de creación y mantenimiento identitario (percepción-acción), y la capacidad de autodiferenciación y devenir (afectividad-emoción). En esta última capacidad se señala una paradoja: el material que detona la diferenciación es siempre carga preindividual, no es propia del individuo sino del resto que guarda en común con lo otro. En otros términos, lo que hace posible que nos vinculemos con otros no es lo que nos identifica sino aquello que no ajusta aún en nuestra identidad, lo que requiere ser completado: de ahí su potencial de innovación.

A partir del reconocimiento de la afectividad y su incidencia en la comprensión de autonomía aquí planteada, se propone una definición del Diseño ontológico transinvididual. Para ir más lejos, plantea la innovación como un proceso que sólo se desata, no por efecto de una subsunción a una lógica heterónoma (colonización), sino cuando la identidad es puesta en entredicho al tiempo que lo otro lo es también, lo cual da lugar a nuevos mundos posibles. Este proceso de transindividuación entre mundos ocurrirá solo por la vía expresiva lo que supone una actividad performativa: Diseño.

Esta propuesta no supone en absoluto el abandono o el no reconocimiento de las autonomías de los diversos movimientos sociales latinoamericanos, al contrario. Estos colectivos brindan otras maneras de ser en el mundo. Lo que es innegable es que tenemos que actuar en comunión. Ni los pueblos por sí mismos lograrán librar la batalla al Capitaloceno y su monstruosa consumición del planeta, ni nosotros lo haremos de permanecer asidos a nuestras viejas prácticas y visiones. De ahí la importancia de un Diseño ontológico-político transindividual. 


\section{Referencias}

Arendt, H. (2009). La condición humana. Editorial Paidós.

Baggs, E. \& Chemero, A. (2018). Radical embodiment in two directions. Synthese.

Beck, U. (1998). La sociedad del riesgo. Hacia una nueva modernidad (J. Navarro, D. Jiménez \& M. Borrás, Trad.). Editorial Paidós. (Obra original publicada en 1986).

Cruz, A. (2018, febrero 23). Pequeñas personas, pequeños haceres. Revista Arquine. https://www.arquine.com/ pequenas-personas-pequenos-haceres/

Cruz A. \& Cortés E. (2020). Design for Inclusion: From Ethical Through Aesthetic Thinking, en ICDHS 12: Lessons to Learn? Past Design Experiences and Contemporary Design Practices Proceedings. ICDHS.

Deleuze, G. \& Guattari, F. (2001). ¿Qué es Filosofía? (T. Kauf, Trad.). Anagrama. (Obra original publicada en 1991)

Deleuze, G. \& Guattari, F. (2002). Mil Mesetas. Capitalismo y Esquizofrenia (J. Vázquez, Trad.). Pre-textos. (Obra original publicada en 1988).

Escobar, A. (2014). Sentipensar con la tierra. Nuevas lecturas sobre desarrollo, territorio y diferencia. Ediciones UNAULA.

Escobar, A. (2016). Autonomía y diseño. La realización de lo comunal. Editorial Universidad del Cauca.

Escobar, A. (2017). Response: Design for/by [and from] the 'global South'. Design Philosophy Papers, 15(1), 3949. DOI: 10.1080/14487136.2017.1301016

Esteva, G. (2008). Agenda y sentido de los movimientos antisistémicos. POLIS, Revista de la Universidad Bolivariana, (19), o.

Flusser, V. (1999). Filosofía del diseño (P. Marinas, Trad.). La forma de las cosas. Editorial Síntesis. (Obra original publicada en 1993).

Foucault, M. (1988). Nietzsche, la genealogía, la historia (J. Vázquez, Trad.). Pre-textos. (Obra original publicada en 1971).

Fry, A. (2017). Design for/by "The Global South". Design Philosophy Papers, 15(1), 3-37. DOI: $10.1080 / 14487136.2017 .1303242$

Gibson, J. (1979). The ecological approach to visual perception. Houghton Lifflin.
Guattari, F. (1996). Las tres ecologías (J. Vázquez \& U. Larraceleta, Trad.). Pre-textos. (Obra original publicada en 1989).

Hardt, M. \& Negri A. (2004). Multitud. Guerra y democracia en la era del Imperio. Debate.

Heidegger, M. (1986). El sery el tiempo /J. Gaos, Trad.). Fondo de Cultura Económica (Obra original publicada en 1927).

Ingold, T. (2011). Being alive: essays on movement, knowledge, and description. Routledge.

James, W. (1977). A Pluralistic Universe. Harvard University Press (Obra original publicada en 1909).

Jonas, H. (1995). El principio de responsabilidad. Ensayo de una ética para la civilización tecnológica (J. Fernández, Trad.). Herder. (Obra original publicada en 1979).

Kant, I. (2005). Crítica del juicio (J. Rovira, Trad.). Editorial Losada. (Obra original publicada en 1790).

Kuhn, T. (1989). ¿Qué son las revoluciones científicas? (J. Romo, Trad.). Ediciones Paidós. (Obra original publicada en 1987).

Maturana, H. \& Varela, F. (1980). Autopoiesis and cognition: the realization of the living. Reidel Publishing Company. Maturana, H. \& Varela, F. (1987). The tree of knowledge: the biological roots of human understanding. Shambhala.

Rocha, M. (2015). Oralitographic Textualities and Head-up Visions in Contemporary Indigenous Oraliteratures in Colombia. PhD dissertation, Department of Romance Studies, University of North Carolina.

Rolnik, S. (2006). Antropofagia Zombie, en Arte, máquinas, trabajo inmaterial. Brumaria.

Rossi, P. (2015). Del pluriverso al cosmograma. Ontología, política y tecnociencia. XI Jornadas de Sociología. Facultad de Ciencias Sociales, Universidad de Buenos Aires, Buenos Aires.

Santos, B. (2009). Una epistemología del sur: la reinvención del conocimiento y la emancipación social. Siglo XXI y CLACSO.

Savransky, M. (2019). Pensar el Pluriverso: Elementos para una Filosofía Empírica. Revista Diferencias, 8, 62-71.

Simondon, G. (2015). La individuación a la luz de las nociones de forma y de información (P.Ires Trad.). Editorial Cactus (Obra original publicada en 1964).

Winograd, T. \& Flores, F. (1989). Hacia la comprensión de la informática y la cognición. Editorial Hispano Europea. 